\title{
EchoGéo
}

39 | 2017

Ressources urbaines (1)

\section{De par la ville}

Jean-Louis Chaléard

\section{(2) OpenEdition}

Journals

Electronic version

URL: https://journals.openedition.org/echogeo/14926

DOI: $10.4000 /$ echogeo.14926

ISSN: 1963-1197

\section{Publisher}

Pôle de recherche pour l'organisation et la diffusion de l'information géographique (CNRS UMR 8586)

\section{Electronic reference}

Jean-Louis Chaléard, "De par la ville", EchoGéo [Online], 39 | 2017, Online since 28 March 2017, connection on 10 August 2021. URL: http://journals.openedition.org/echogeo/14926 ; DOI: https:// doi.org/10.4000/echogeo.14926

This text was automatically generated on 10 August 2021

EchoGéo est mis à disposition selon les termes de la licence Creative Commons Attribution - Pas d'Utilisation Commerciale - Pas de Modification 4.0 International (CC BY-NC-ND) 


\title{
De par la ville
}

\author{
Jean-Louis Chaléard
}

1 La nouvelle livraison d'Échogéo est largement consacrée à des questions portant sur la façon d'approcher ou d'appréhender la ville. Le dossier Sur le champ le fait à travers une interrogation sur les "ressources urbaines». La rubrique Sur le métier apporte une réflexion sur la géographie urbaine critique, tandis que la rubrique Sur l'écrit nous propose une nouvelle façon de lire la ville à travers un atlas informatique. L'ensemble nous montre la rapidité et l'ampleur des changements concernant aussi bien l'analyse des espaces urbains que des outils permettant de les appréhender. Les thèmes abordés touchent à des questions très débattues actuellement, comme en témoigne le succès de l'appel à communications pour ce dossier sur les ressources urbaines. Devant le nombre élevé de communications retenues, nous avons décidé de les répartir sur deux numéros : ce n'est donc que la moitié des articles sur le thème que propose ce numéro 39 d'Échogéo.

2 Le dossier Sur le champ dirigé par A. Michel et A. Ribardière s'intéresse aux « ressources urbaines » en elles-mêmes, mais aussi comme analyseur de la société et de l'espace urbains. Un large éventail de ressources est pris en compte (logement, emploi, école, santé, transport, etc.), permettant aux auteures, dans leur introduction, de préciser la notion de ressources urbaines. Les approches sont variées, même si les approches qualitatives sont les plus nombreuses, fruit d'un travail souvent minutieux de terrain, qui fournit une masse importante de données de première main sur lesquelles s'appuient les études. Les différentes communications font apparaitre les ressources comme des marqueurs des écarts socio-spatiaux dans la ville, à la fois en raison de leur répartition ou accès inégal, mais aussi de l'inégale capacité de chacun à les mobiliser ou à en mobiliser certaines. Elles les inscrivent aussi dans la durée, mettant en évidence le poids des permanences, des héritages et les nouveaux agencements de la fabrique urbaine.

3 Si chaque article insiste sur un ou quelques types de ressources, des récurrences apparaissent, soulignant de grandes tendances : importance des mobilités au sein de la ville comme ressource et moyen d'accès à d'autres ressources, poids de l'informel dans les grandes agglomérations des Suds, etc. La réflexion s'articule cependant autour de 
quelques questions principales que les responsables du dossier ont organisées en quatre thèmes, dont deux sont présentés dans ce numéro d'EchoGéo. Les trois premiers textes s'interrogent sur la répartition des ressources dans la ville, ou plus précisément dans les périphéries. Ils mettent en évidence concentration hétérogène des ressources selon les espaces et la diversité des façons dont ces derniers s'articulent au reste de la ville. E. V. Cerqueira s'intéresse aux inégalités d'accès aux ressources urbaines dans les franges périphériques de Belo Horizonte, montrant la dépendance des plus pauvres visà-vis du centre, et la reproduction en périphérie des inégalités sociales. J.-F. Valette, qui étudie les colonies populaires à la périphérie de Mexico, souligne l'existence de plusieurs types de rapports à l'espace urbain et la diversité des ancrages locaux. Dans l'ensemble, les colonies populaires sont plus articulées au reste de la métropole qu'on ne pourrait le penser et l'ancrage des populations dans leurs quartiers ne semble pas aller de soi. V. Berdoulay et Thi Thu Trang Ngo étudient les changements dans les rapports sociaux de sexe dans trois types d'habitat à la périphérie d'Ho Chi Min ville. Ils analysent les similarités et les différences à propos des représentations du rôle et de la place des femmes dans la famille et la société et de certaines pratiques. Celles-ci se traduisent dans l'accès aux ressources urbaines potentiellement utilisables.

Les trois textes suivants portent sur les pratiques de la ville par des acteurs au statut de citadin ou de citoyen peu consolidé voire précaire (migrants, habitants des favelas...). Ils montrent que ces acteurs n'ont à leur disposition que les ressources fournies par la ville (diversité des fonctions et des lieux, interface dans la circulation...) et restent en situation exposée et vulnérable. S. Souchaud s'intéresse au cas des migrants boliviens et paraguayens qui travaillent dans le secteur de la confection à Sao Paulo. Le modèle d'organisation des ateliers montre que les ressources urbaines relèvent autant d'un usage (réaffectation de bâtiments dévalorisés mais en position centrale au sein de la métropole) que d'une production (logement pour les migrants). De façon ambivalente, les ateliers sont une ressource pour les migrants et favorisent leur insertion urbaine, en même temps qu'ils permettent de capter une main-d'œuvre immigrée faiblement rémunérée par les patrons. L. Faret prolonge l'interrogation sur la mobilisation des ressources urbaines par les migrants en s'intéressant aux rapports entre trajectoires de mobilité et installation dans l'espace urbain par les Centraméricains à Mexico. L'attractivité de Mexico est réelle, mais les parcours des migrants sont aussi le résultat de trajectoires bloquées, autant vers le Nord (États-Unis...) que vers le Sud (retour au pays). Ces populations construisent alors des stratégies d'installation et d'insertion socio-économique dans un contexte de forte précarité, s'adressant aux associations et s'appuyant sur les caractéristiques mêmes de la métropole (notamment l'existence de centralités secondaires, source de connaissances voire d'emplois). Dans le cas de Rio de Janeiro, R. Gonçalves montre que la situation géographique et matérielle des favelas peut constituer une ressource urbaine. Celles-ci offrent un habitat en situation centrale dans la ville à un prix abordable pour les populations démunies et des logements plus adaptés aux besoins des résidents. Ainsi, les six textes publiés dans ce numéro d'ÉchoGéo permettent-ils une première approche de la question des ressources urbaines, soulignant la complexité des situations, l'ambivalence fréquente des ressources et leur rôle dans la survie des populations précaires. La suite, publiée en juin dans le numéro 40 d'ÉchoGéo, complètera et enrichira le sujet.

5 Il est encore question de ville dans la rubrique Sur le métier, mais l'approche et le contenu sont différents du dossier Sur le champ. C. Gintrac analyse «la fabrique de la géographie urbaine critique et radicale ». À partir de l'étude de deux groupes au Brésil, 
en Allemagne, et d'un réseau international dont le centre est en Suisse, elle essaie de dégager les traits concordants d'une géographie urbaine critique: des fondements théoriques marxistes et postmarxistes s'appuyant sur un référentiel commun d'auteurs (Harvey, Lefebvre, Smith, Soja...) ; une grille d'analyse centrée sur la critique des formes de domination et les conditions de production de la recherche; un dépassement de la spécialisation des savoirs et de la coupure entre le monde universitaire, le monde militant et le monde social, qui débouchent sur un engagement dans les mouvements sociaux. L'originalité de cette approche se situe donc autant au niveau théorique que dans ses conséquences sur les pratiques de ses tenants.

6 La rubrique Sur l'écrit présente un nouvel atlas en ligne: l'Atlas social d'Athènes. C'est l'occasion pour S. Weber d'interroger un des auteurs, S. Spyrellis, et de mettre en évidence la richesse des fonctionnalités et la souplesse d'utilisation de cette nouvelle forme de production: diversité des échelles abordées (de la rue à l'agglomération), originalité des sujets traités (avec une entrée sur l'austérité par exemple), etc. Se présentant comme une ressource ouverte, l'atlas est fait pour des publics variés, scientifique et plus large (touristes, habitants...). L'atlas en ligne n'est ainsi pas seulement un nouveau produit technique, issu des ressources informatiques. Il permet d'innover au plan du contenu scientifique et de la diffusion des savoirs. C'est une voie que la géographie se doit de creuser. 